\title{
Inovando o Trabalho de Campo com a Articulação entre Teoria e Prática: as Disciplinas do Curso de Obstetrícia
}

\author{
Eunice Almeida da Silva, Nádia Zanon Narchi, Maria Aparecida de Jesus Belli, Maryam \\ Michelle Jarrouge Trintinália, Nilva Teixeira \\ Escola de Artes, Ciências e Humanidades da Universidade de São Paulo \\ * Autora para correspondência: eunice.almeida@usp.br
}

\begin{abstract}
RESUMO
Este estudo tem como finalidade mostrar a estratégia de ensino utilizada nas disciplinas Administração dos Serviços de Atenção à Saúde e Estágio Curricular de Administração de Serviços de Atenção à Saúde, obrigatórias no curso de graduação em Obstetrícia da Universidade de São Paulo. O objetivo geral da estratégia foi articular o conteúdo oferecido em sala de aula às práticas em gerenciamento. Em 2014, 43 alunos cursaram as disciplinas, discutiram o conteúdo programático em sala de aula, realizaram visitas a serviços da rede privada e vivenciaram práticas de gerenciamento em serviços públicos de saúde. O objetivo específico era identificar e analisar até três situações-problema que poderiam dificultar a dinâmica de gerenciamento dos serviços públicos de saúde. A partir da análise e com base nas visitas realizadas, os alunos em grupos elaboraram um projeto visando à melhoria do gerenciamento dos serviços públicos. Os projetos deveriam responder a questão: "É possível administrar os processos, os recursos e as pessoas nos serviços públicos de atenção à saúde de maneira a agregar-lhes valor?" Todos responderam afirmativamente, evidenciando descrições de como foram compreendidas as articulações dos conceitos discutidos em sala de aula com as dificuldades e possibilidades encontradas nos cenários das práticas.
\end{abstract}

Palavras-chave: Ensino; Gerência de Serviços de Saúde; Teoria; Prática Profissional; Obstetrícia.

\begin{abstract}
This study aims to show the teaching strategy used in the disciplines Administration of Health Care Services and Internship of Administration of Health Care Services, mandatory in the undergraduate course in Obstetrics of the Universidade de São Paulo. The general objective of the strategy was to articulate the content offered in the classroom to the internship practice in management. In 2014, forty-three students attended the subjects, discussed the content in the classroom, carried out visits in the private network services and experienced management practices in health public services. The specific objective of the strategy was to identify and to analyze three problem situations that could hinder the dynamic management of health public services. From the analysis and based on the visits, students in groups drew up a project aimed at improving the management of public services. Projects should answer the question: Is it possible to manage the processes, resources and workers in public health care services in order to add value to them? All answered affirmatively, showing descriptions of how the connections between the concepts discussed in class with the difficulties and possibilities found in the scenarios of the practices were understood.
\end{abstract}

Keywords: Teaching; Health Service Management; Theory; Professional Practice; Obstetrics.

\section{Introdução}

Este estudo tem como objetivo central apresentar uma estratégia de ensino pautada no conteúdo das disciplinas Administração dos Serviços de Atenção à Saúde e Estágio Curricular de Administração de Serviços de Atenção à Saúde, obrigatórias para os que cursam o oitavo período da graduação em Obstetrícia na Escola de Artes, Ciências e Humanidades da Universidade de São Paulo.

O curso de graduação em Obstetrícia é oferecido no período diurno, em nove semestres letivos, com carga horária mínima de 4350 horas, e 
com estrutura curricular organizada de maneira a "formar profissionais para atuar na educação e na promoção da saúde das mulheres, das famílias e da comunidade, com foco especial na atenção à maternidade e no gerenciamento do cuidado e de serviços de atenção à saúde das mulheres" (EACH-USP, 2016).

O curso de graduação em Obstetrícia é formado por três eixos: 1- eixo de bases biológicas; 2- eixo de ciências humanas, sociais e da saúde e 3- eixo assistir, cuidar e gerenciar. As disciplinas de Administração se inserem no terceiro eixo.

$\mathrm{O}$ ensino de gerenciamento do cuidado e de serviços para os graduandos em Obstetrícia se faz de maneira integrada. Busca-se discutir a trajetória de teorias e conceitos em Administração e a partir desses relacioná-los com teorias e conceitos próprios da área da saúde, em particular do campo da Obstetrícia.

Em 2014 a disciplina Administração dos Serviços de Atenção à Saúde, cuja carga horária foi de sessenta horas, teve como objetivo principal apresentar conceitos básicos da Teoria Geral da Administração (TGA) e suas correlações com os diferentes modelos de gestão em saúde. O conteúdo programático abarcou as seguintes temáticas: noções gerais da Teoria Geral da Administração; cultura e poder nas organizações; competências e habilidades do administrador; modelos de planejamento e gestão de modelos de atenção em obstetrícia; gestão de recursos, processos e pessoas; avaliação de serviços de saúde. Em gestão de recursos, as discussões abarcaram os recursos físicos e ambientais, bem como os recursos materiais e financeiros. Em gestão de processos o enfoque foi dado para normas, fluxos, rotinas e procedimentos; em gestão de pessoas foram oferecidas as temáticas: histórico de gestão de pessoal e das relações de trabalho nos serviços de atenção à saúde; gestão estratégica de recursos humanos; recrutamento e seleção; formação profissional, desenvolvimento e treinamento; comunicação e informação; liderança; motivação; assédio moral; gerenciamento de conflitos e negociação; tomada de decisão; avaliação e performance profissional/marketing pessoal; qualidade de vida e gestão de pessoas.
A disciplina Estágio Curricular de Administração de Serviços de Atenção à Saúde, com carga horária de noventa horas, teve como objetivo central possibilitar a vivência em diferentes cenários das práticas de gerenciamento nos serviços de saúde. O conteúdo programático abarcou os seguintes temas: gerenciamento do cuidado/gestão de modelos de atenção em obstetrícia; processo gerencial em recursos humanos: dimensionamento, recrutamento, seleção, educação permanente, tipos de avaliação, liderança, supervisão e motivação, comunicação e informação nas relações profissionais. A disciplina pautou-se pelo desenvolvimento de atividades teórico-práticas no gerenciamento de serviços de atenção à saúde da mulher e à saúde materna e perinatal.

Um professor doutor ficou responsável pela disciplina Administração dos Serviços de Atenção à Saúde. A organização da disciplina Estágio Curricular de Administração de Serviços de Atenção à Saúde incluiu três professores doutores e três especialistas que auxiliaram a supervisionar os campos de estágio.

A principal meta da prática era articular o embasamento teórico discutido na disciplina Administração dos Serviços de Atenção à Saúde com as questões levantadas nas visitas aos serviços privados, filantrópicos de saúde e na vivência nos serviços públicos. O resultado deveria produzir ideias de como seria possível administrar os processos, os recursos e as pessoas nos serviços públicos de atenção à saúde de maneira a agregar-lhes valor.

\section{Descrição da Estratégia de Ensino}

Os 43 graduandos, que cursaram as disciplinas referidas no segundo semestre de 2014, foram divididos em duas equipes, subdivididas em três subequipes. As subequipes visitaram dois grupos de serviços de saúde. O grupo A foi composto por dois hospitais públicos situados na Zona Leste de cidade de São Paulo e um hospital público localizado no município de Ferraz de Vasconcelos. Todos oferecem serviços a mulheres gestantes.

O Grupo B foi composto por quatro serviços: uma casa de parto de caráter filantrópico, localizada na Zona Leste da cidade de São Paulo; uma 
unidade de tratamento de resíduos sólidos de saúde do setor privado, situada na Zona Oeste da cidade de São Paulo; um hospital escola público de grande porte, localizado na Zona Oeste de São Paulo; um hospital público com serviços destinados exclusivamente a mulheres, situado na região metropolitana de São Paulo.

As visitas realizadas pelas subequipes tinham como principal objetivo identificar e analisar até três situações-problema que poderiam dificultar a dinâmica de gerenciamento dos serviços públicos de saúde. As análises produzidas pelos alunos em conjunto com os conhecimentos teóricos tecidos na disciplina Administração dos Serviços de Atenção à Saúde serviram de subsídio para a elaboração de um projeto de melhoria do gerenciamento dos hospitais públicos.

Todos os projetos deveriam responder a questão central: É possível administrar os processos, os recursos e as pessoas nos serviços públicos de atenção à saúde de maneira a agregar-lhes valor?

Foram elaborados oito projetos e selecionados seis com base naqueles que responderam a questão central. As situações-problema identificadas e analisadas trataram das temáticas: motivação profissional; resíduo sólido hospitalar; alojamento conjunto; modelos de planejamento; centro obstétrico e liderança.

\section{Discussão e Revisão da Literatura}

A temática sobre a desarticulação entre teoria e prática tem sido explorada de maneira exaustiva na área da Educação e, em particular, na área da Saúde. Reconhecidamente é um tema que abarca uma enorme complexidade, pela dificuldade de harmonizar essas duas facetas (ALVARÉZ, 2012; SILVA, 2013).

A formação do profissional em saúde inclui, geralmente, o estágio curricular nos cenários das práticas em hospitais, Unidades Básicas de Saúde (UBS), clínicas, dentre outros. A prática articulada à formação do estudante é reconhecidamente importante por possibilitar a sua vivência no mercado de trabalho em que futuramente irá se inserir. Apesar disso, o contato do estudante com as práticas pode causar sofrimento e conflitos capazes de dificultar o processo de ensino-aprendizado (MOULIN, 1998). Essa situação poderia ser atribuída à desarticulação entre a teoria e a prática, ou seja, o que é discutido em sala de aula nem sempre se contempla nos cenários das práticas, provocando grandes frustrações tanto para os estudantes quanto para os supervisores.

Muitos são os estudos que apontam para essa questão. Alguns trabalhos, como o de Silva e Romanini (2014), Alvaréz (2012), Schon (2009), Bagnato e Sordi (2007), Bellato et al. (1997), Enders et al. (1995), Lima (1994) e Argyris e Schon (1992), atribuem a desarticulação entre teoria e prática a contradições existentes no ensino e na atuação em serviços.

Scherer et al. (2006) fazem um estudo que mostra a reação do aluno diante das primeiras experiências oferecidas pela profissão: a ansiedade e a angústia sentidas. As autoras atribuem esses sentimentos ao contexto pessoal, social e ambiental, mas também à discrepância entre o que é dito na teoria e o que acontece na prática.

O estudo de Ribeiro (2006) aponta a importância de serem desenvolvidos trabalhos que busquem avaliar determinantes da qualidade e do aproveitamento de estágios na área da saúde. Segundo o autor, esses estudos poderiam revelar possibilidades de resoluções para problemas relacionados com os sistemas de serviços de saúde. Isso poderia facilitar a compreensão e o significado das atividades desenvolvidas nos serviços, retratando a dinâmica das equipes de saúde.

Outros trabalhos, como os de Rudnicki e Carlotto (2007), Comas Vargas e Ariza Theran (2007) e Nogueira-Martins (2002), mostram as reações dos estudantes em campo de estágio quando se deparam com as limitações relativas ao distanciamento entre a teoria discutida em sala de aula e as ações práticas. Segundo os autores, essa situação é decorrente também das discussões superficiais que acontecem na sala de aula, em que os problemas ocorridos nos cenários das práticas não são abordados ou o são de maneira superficial.

Rozada (2007) estuda algumas condições fundamentais para facilitar a articulação entre a teoria 
e a prática; uma delas é respeitar a importância da teoria e da prática separadamente. A partir daí, nem a teoria está acima da prática e nem a prática acima da teoria. Busca-se, entretanto, estabelecer uma relação dialética e dialógica entre ambas.

\section{Considerações Finais}

A estratégia pedagógica utilizada para entrelaçar as duas disciplinas, Administração dos Serviços de Atenção à Saúde e Estágio Curricular de Administração de Serviços de Atenção à Saúde, contribui para a formação de profissionais, uma vez que os capacita a minimizarem as contradições identificadas entre o ensino e as ações realizadas nos serviços, enfaticamente apontadas nos estudos citados anteriormente. Colabora também para estimular reflexões sobre a importância da articulação entre o que é dito em sala de aula e as necessidades expressas nos campos de trabalho. Além disso, estimula os estudantes a planejarem possibilidades de ressignificar a dinâmica do trabalho na área da saúde, a qual ainda se organiza de forma fragmentada, o que repercute na qualidade do trabalho e da assistência em saúde, por mais que existam esforços por parte dos profissionais e órgãos governamentais para reduzir/eliminar o fazer mecanizado.

A questão central dos projetos que os alunos elaboraram, "É possível administrar os processos, os recursos e as pessoas nos serviços públicos de atenção à saúde de maneira a agregar-lhes valor?", foi respondida afirmativamente; as indicações de melhorias para os hospitais públicos envolvidos foram embasadas teoricamente e justificadas pelas experiências vivenciadas nos serviços visitados. Os projetos trouxeram descrições de como foram elaboradas as articulações entre os conceitos discutidos na disciplina Administração dos Serviços de Atenção à Saúde e os eventos que surgiram nos cenários das práticas.

Esta experiência mostrou-se viável e criativa para o processo de ensino-aprendizagem, pontuando a estratégia pautada no ensino baseado em resolução de problemas, além de relevar a importância da integração entre ensino, pesquisa e extensão.

\section{Referências Bibliográficas}

ÁlvarEZ, C. A. "¿Qué Sabemos de la Relación entre la Teoría y la Práctica en la Educación?". Revista Iberoamericana de Educación Organización de Estados Iberoamericanos para la Educación, la Ciencia y la Cultura (OEI-CAEU), vol. 60, n. 2, pp. 1-11, 2012.

ARGYRIS, C. \& SCHON, D. A. Theory in Practice: Increasing Professional Effectiveness. San Francisco: Jossey-Bass, 1992.

BAGNATO, M. H. S. \& RODRIGUES, R. M. "Diretrizes Curriculares da Graduação de Enfermagem: Pensando Contextos, Mudanças e Perspectivas". Revista Brasileira de Enfermagem, vol. 60, n. 5, p. 510, set.-out. 2007.

BELLATO, R.; PASTI, M. J. \& TAKEDA, E. "Algumas Reflexões sobre o Método Funcional no Trabalho da Enfermagem". Rev. Latino-Am. Enfermagem, Ribeirão Preto, vol.5,n.1,pp.75-81,jan. 1997.Disponível em: <http://www.scielo.br/scielo.php?script=sci arttext\&pid=S0104-11691997000100009\&lng=en\&nrm =iso $>$. Acessado em 7 maio 2017.

CARLOTTO, M. S. \& RUDNICKI, T. "Formação de Estudante da Área da Saúde: Reflexões sobre a Prática de Estágio". Rev. SBPH, Rio de Janeiro, vol. 10, n. 1, pp.7-110, jun. 2007.

EACH-USP-2016. Disponível em: <http://www5. each.usp.br/wp-content/uploads/2015/11/Projeto-Pedag\%C3\%B3gico-do-Curso-de-Obstetricia-2016.pd† $>$. Acessado em 31 mar. 2016.

LIMA, M. A. D. S. "A Formação do Enfermeiro e a Prática Profissional: Qual a Relação?", Rev. Gaúcha Enf., vol. 15, n. 1/2, pp. 34-40, jan.-dez. 1994.

MOULIN, M. G. B. "Trabalho, Saúde Mental e Gênero: O Caso das Bancárias". F. Bras. Psiq., vol. 47, n. 4, pp. 169-177.

RIBEIRO, S. F. R. Sofrimento Psíquico dos Trabalhadores de uma Equipe do Programa Saúde da Família na Organização do Trabalho. Dissertação (Mestrado), Faculdade de Medicina de Botucatu, Universidade Estadual Paulista, 2006. 162p.

ROZADA, J. M. Formarse como Profesor. Ciencias Sociales, Primaria y Secundaria Obligatoria (Guía de Textos para un Enfoque Crítico). Madrid: Akal, 1997.

SCHERER, Z. A. P.; SCHERER, E. A. \& CARVALHO, M. P. "Reflexões sobre o Ensino da Enfermagem e os Primeiros Contatos do Aluno com a Profissão", Rev. Latino-Am. Enfermagem, vol. 14, n. 2, pp. 285-91, mar.-abr. 2006.

SCHON, D. A. Educating the Reflective Practitioner. New York: Basic Books, 2009.

SILVA, E. A. A Relação Teoria-Prática em um Curso de Pós-Graduação em Enfermagem. Relatório de pesquisa (Pós-doutorado em Educaşão), Faculdade de Educaşão, 
Universidade Estadual de Campinas, Campinas-Brasil, 2013. fissional de Saúde na Perspectiva da Semiótica”. Cadernos \& ROMANINI, A. V. "A Formação do Pro- ～de Semiótica Aplicada, vol. 12, n. 2, pp. 103-127, 2014.

Publicado em 30/06/2017. 